\title{
An Application of a Low-Cost Monitoring and Guidance System in a WSN
}

\author{
Yetkin TATAR \\ Assoc.Prof. \\ Department of Computer Eng., Firat University, \\ Elazig, Turkey
}

\author{
Güngör YILDIRIM \\ Electrical - Electronics Engineer \\ DSI IX. Regional Directorate, \\ Elazig, Turkey
}

\begin{abstract}
Wireless sensor networks (WSNs) have been deployed in many workplaces (hangars, warehouses, etc.) for general purposes (climatic data collection, security and so on). Also, in these kinds of workplaces, localization systems are often required for monitoring, tracking and guidance, but this means extra charge. In this article, an application of a low-cost localization system, which can track and guide vehicles by the help of an existing WSN system, is introduced. For the system, a technique called "Edge-Masking Technique" was proposed. Besides, "Bounding Box" method was used for the position detection. The empiric RSSI-distance relationship equation used in the method was obtained from experimental studies. In addition to this, the equation was improved by a technique named "Circular Calibration with Offset Value". The practicability of the system was demonstrated by both simulation and real nodes.
\end{abstract}

\section{Keywords}

Wireless Sensor Networks, Position Detection, TinyOS, TOSSIM, Min-Max Method.

\section{INTRODUCTION}

Wireless sensor network (WSN) is an ad-hoc network that has a lot of sensor nodes which are able to communicate each other according to the 802.15.4 network protocol in a media with radio frequency (RF) [1][2][5][14]. In recent years, WSNs have received important attention. This technology has been used in many industrial applications because of dynamic features. WSNs include numerous research fields. One of the fields is a localization issue, especially, in indoor places in which GPS is insufficient. The meaning of location detection or position detection in a closed environment is that coordinates of a sensor node in the WSN is detected approximately. There are generally two main methods for position detection based on distance information, "Trilateration" and "Triangulation" [6] The methods need at least three distance values obtained from three anchor nodes whose coordinates known [3][4]. Different techniques can be used for distance estimation. Among these techniques, the techniques based on received signal strength indicator (RSSI) are often preferred because of not needing additional equipment and data packets [19].There are many studies about position detection guidance and tracing in WSNs. Some of them are explained as follows.

A study that is able to trace the moving objects (wheelchairs etc.) between the services of a hospital by the help of the nodes which can be mounted on them in a WSN system was carried out by S.J.Kim and et al [7]. In another study, an implementation that can detect multiple moving targets and trace them by distributed algorithm, and its simulation were done by S.Kamat and et al. [8]. A system to watch a process from a load point to an unloading point by a container monitoring system was carried out by S. Mahlknecht and et al.[9]. G.Y.Jin and et al. proposed a dynamic clustering method which enables nodes only around the target nodes for the energy management of the WSN system [10]. In different study, B.Y.Zong and et al. developed a WSN design which can guide a vehicle for parking in a guarded parking by the help of specific nodes [12]. M.Y.I Idris and et al. improved this parking system and made a smart guarded parking management system which can calculate the most appropriate vacant field for a vehicle and inform the driver and a guarded parking officer about it [13]. In another application, S.Lee and et al. designed a WSN system which can find a vacant park field by evaluating the data obtained from ultrasonic or magnetic sensor nodes in a guarded parking. Evaluation operation was done by the help of "Min-Max Method" [16].

As known, today many workplaces (hangars, big store etc.) have made use of WSNs for various applications, for example climatic monitoring, security, and so forth. Besides, in these kinds of workplaces, monitoring and guidance systems are often required. In general, for this, other technologies (such as cameras) are used, but installation and operation of them mean extra charge. In the article, by the help of an existing WSN system, an application of a low-cost localization system, which can track and guide vehicles in a big closed workplace including thousands of goods, is introduced. This kind of system design has not been come across in literature reviews.

For this, a scenario named "An Application of Tracing and Guidance in an Indoor Place" was created. The scenario has two main purposes; the first is to guide quickly the vehicles with a Mobile Node (MON) for the desired goods by the help of other nodes. The second is to trace them from a central office by a computer and to send commands interactively. For the guidance of the vehicles to a target region, a technique called "Edge-Masking" was proposed and implemented. "Bounding Box Method" was preferred for position detection.

The calibration of the empiric equation of RSSI-Distance relationship used in the method was improved by a developed technique, "Circular Calibration with Offset Value" obtained from experimental studies [2].

In the study, MEMSIC TelosB TPR2420 sensor nodes [21], Ubuntu 11.10 operation system, TinyOS 2.1.0 embedded operation system, TOSSIM emulator and Tkinter GUI-toolkit were used. The application software used was prepared by JAVA programming language. 


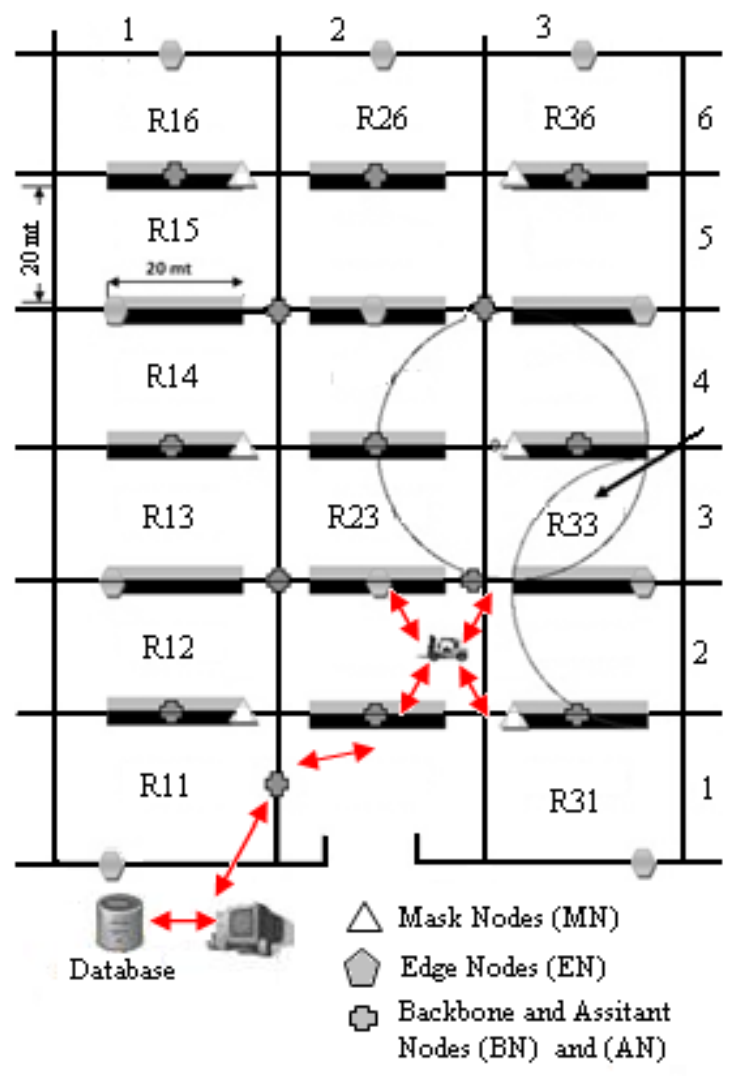

Fig 1: The model of the work area

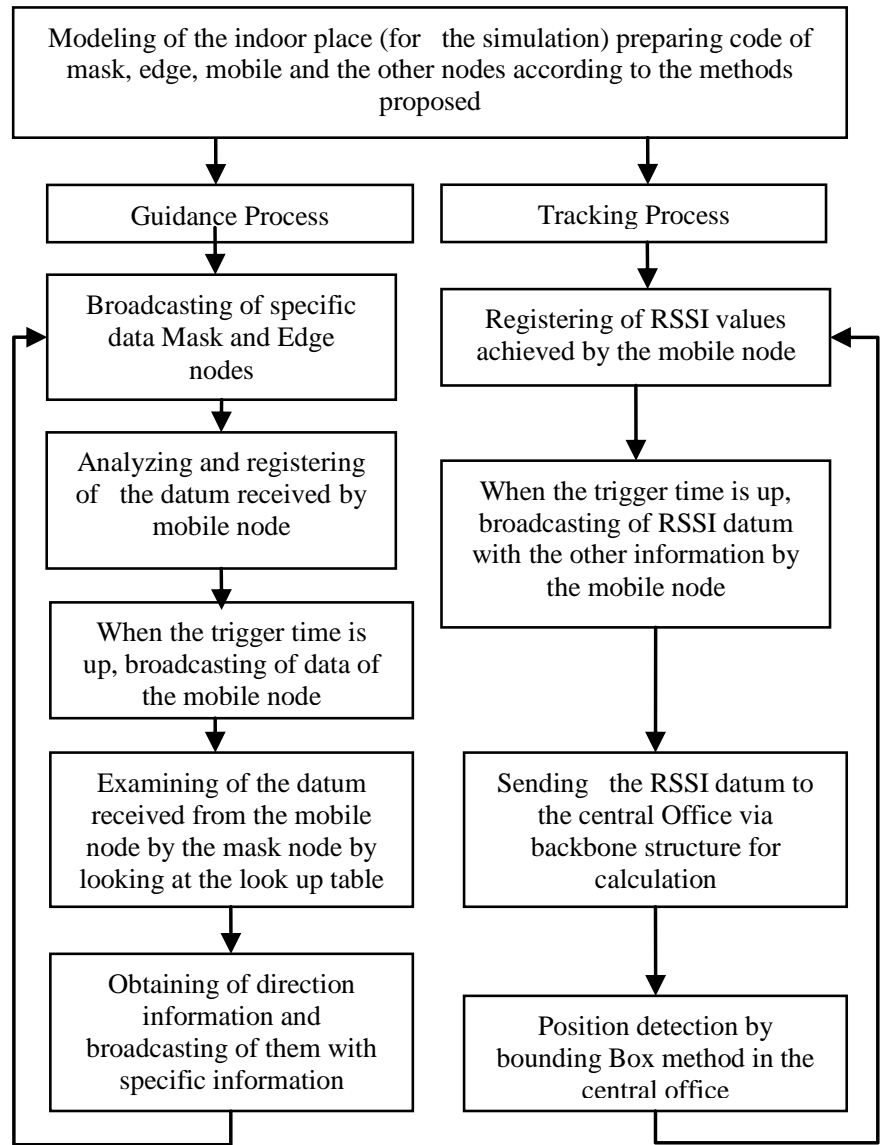

Fig 2) The flow diagram of the all system design

\section{ARCHITECTURE OF THE WSN SYSTEM}

In the scenario, an indoor place which has dimension of 100 $\mathrm{m} \times 120 \mathrm{~m}$ and shelf groups with number was considered. In the Fig. 1, the plan of the indoor workplace, the position of shelf groups and location of the nodes are shown. In order that the guidance of the vehicles was able to be made systematically, the workplace was divided into matrix cells and then the cells were labeled. Thus, the vehicles could be directed easily to the target place according to cell codes (region codes) of the desired goods. In the WSN system, there are four types of nodes, Mask Nodes (MN), Edge Nodes (EN), Backbone Nodes (BN) and Assistant Nodes (AN). At the same time, it is deemed that the nodes have been used for climatic measurements, security etc. The mask and edge nodes were used for region determination, the backbone nodes were used for the communication between the workplace and the central office, the last was used for the distance estimation based on RSSI and position detection. The flow diagram of the all system design is shown in Fig.2.

\subsection{Mathematical Model of the Workplace}

For the simulation of the system, the all workplace architecture described in Fig.1 should be modeled according to reference values. In order to model the area, the upper left corner was marked $(0,0)$ and the 18 cells of $20 \times 30 \mathrm{~m}$ were represented as 100 pixel x 150 pixel. Each matrix cell was labeled as "Rij". There, while "i" index represents vertical code, "j" represents horizontal code.

\subsection{The Edge-Masking Method}

As can be seen from Fig.3, the coverage area of a node could reach many regions (cells). This is a problem for the vehicles to be directed. The location of mobile vehicles (forklift, trucker etc.) has to be known clearly to direct them to the desired region. For the solution of this problem, a technique named "Edge-Masking", based on information obtained from at least two nodes having different operation-mode, was proposed. The technique guarantees more clearly localization in common coverage area created by many nodes.

In Fig. 3, the node "MN" is able to detect whether the vehicle is in its coverage area by communicating with the MON. But it is not able to distinguish exactly what part of the coverage area the vehicle is in. On the other hand, if the coverage area of another node cuts appropriately the coverage area of the $\mathrm{MN}$ and the MON could transmit this intersection-information to the $\mathrm{MN}$, then the $\mathrm{MN}$ will be able to analyze the information coming from the MON by looking at its look-up table. If the intersection-information is defined in the table, the MN will be able to detect clearly which part of the coverage area the vehicle is in. 


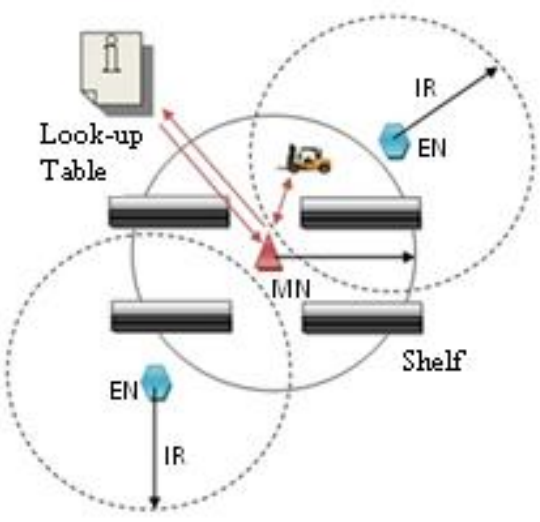

Fig 3: Detection with multiple nodes

In this technique, sizing process of regions is very important. While making the sizing, the indoor range (IR) of nodes has to be considered. The width and height values of the regions should not be smaller than the indoor range of the node. Eq.1 has been explained this rule, there, " $\mathrm{h}_{\mathrm{u}}$ " is the height of the region (cell), " $\mathrm{h}_{\mathrm{g}}$ " is the width of the region and " $\mathrm{d}_{\mathrm{r}}$ " is the indoor range of the node.

$h_{g} \geq d_{y}$

$h_{u} \geq d_{y}$

In the study, the indoor range was chosen $20 \mathrm{~m}$ (from datasheets of micaZ, TelosB), which this value can vary according to technology used. As the size of the regions was specified, an area in which big and medium-sized work vehicles (trucks, 18 wheels, forklifts etc.) were able to maneuver together were considered.

The guidance of the vehicles was carried out by the EdgeMasking Technique. In this technique, each of nodes was classified according to its operation mode. Thus a node has a "node-type number" as well as "node ID". For example, while mask-nodes have a node-type number "2", edge-nodes have node-type number " 3 ". The numbers help the WSN system expand flexibly. While passing through a region, the MON collects data packets and analyze them according to node-types. If the MON receives a data packet from a node which has a mask node-type number, it will record the node ID of the MN in its memory, and similarly, if it receives a data packet from a node which has an edge node-type number, it will record the node ID of the EN. Besides, for the position detection of the MON, the RSSI values have to be recorded in the memory. When the trigger time of the MON is expired, it will broadcast a data packet including its node ID, the nodetype number, mask-node ID recorded, edge-node ID recorded, the RSSI-values recorded (RS), the vertical and horizontal codes of the target region $(n, m)$. The payload is shown in the Table I.

Table 1. A payload structure the MON broadcasts

\begin{tabular}{|c|c|c|c|c|c|c|c|c|}
\hline $\begin{array}{c}\text { Nod } \\
\text { ID }\end{array}$ & $\begin{array}{c}\text { Nod } \\
\text { Type }\end{array}$ & $\begin{array}{c}\text { Mask } \\
\text { ID }\end{array}$ & $\begin{array}{c}\text { Edge } \\
\text { ID }\end{array}$ & $\mathrm{n}$ & $\mathrm{m}$ & $\mathrm{RS} 1$ & $\mathrm{RS} 2$ & $\mathrm{RS} 3$ \\
\hline
\end{tabular}

The MN receiving the data packet from the MON will examine mask-node ID, edge-node ID, vertical code (n) and horizontal code $(\mathrm{m})$ values by the help of its look-up table. If the values are defined in the table, the MN will distinguish which part of the coverage area the MON is in. For example, if the MON has mask-node ID $=9$ and edge-node ID $=7$, the $\mathrm{MN}$ receiving this data packet will find that the MON is in the region "R23" by looking the Table 2 .

Table 2. The Look-Up Table for Node 9

\begin{tabular}{|c|c|c|}
\hline Mask ID & Edge ID & (Rij) \\
\hline 9 & 6 & R33 \\
\hline 9 & 7 & R23 \\
\hline 9 & 11 & R34 \\
\hline 9 & 12 & R24 \\
\hline
\end{tabular}

$$
\begin{aligned}
& (i=n) \cap(j=m) \rightarrow \text { The target region }{ }_{x} B_{i j} \\
& (i=n) \cap(j<m) \rightarrow \text { Move up } \\
& (i=n) \cap(j>m) \rightarrow \text { Move Down } \\
& (i=n) \cap(j=m) \rightarrow \text { Move horizontal }
\end{aligned}
$$

After the MN makes necessary controls according to Eq. 2, sends the appropriate direction information to the MON. The packet sent has the information shown in Table 3. The packet includes mask-node ID, node-type, "Ok" field to represent the arrival information, the direction fields (Up, Down, Go Straight) and the vertical code (n) for horizontal movement.

Table 3. The packet MN sends to the MON

\begin{tabular}{|c|c|c|c|c|c|c|}
\hline $\begin{array}{c}\text { Nod } \\
\text { ID }\end{array}$ & $\begin{array}{c}\text { Nod } \\
\text { Type }\end{array}$ & Ok & Up & Down & $\begin{array}{c}\text { Hor } \\
\text { Val }\end{array}$ & $\begin{array}{c}\text { Ver. } \\
\text { val }\end{array}$ \\
\hline
\end{tabular}

The strategy of the system is that a region should receive signals from at least two different nodes, which one of them has to be a MN. The coverage areas of the MNs must not intersect a lot of $\mathrm{MN}$ signals because this causes to increase complexity. Besides, although several EN signals can intersect in a region, a minimum number of EN should be used for a small look-up table. Therefore, different approaches and optimization techniques could be preferred for the placement of nodes. Also, before the system is designed, it will be better that a signal distribution of the area is known.

\subsection{Tracing of Mobile Nodes}

The instant position knowledge of the vehicles is necessary for tracing from a central office. In the study, Bounding Box (Min-Max Method) method, based on RSSI-Distance relationship, was preferred for the position detection [3]. Fig 4 explains the method geometrically. There, while MD represents the target node, $\mathrm{C} 1, \mathrm{C} 2, \mathrm{C} 3$ represent the anchor nodes. If the distance "dij", between the target node " $\mathrm{i}$ " and anchor node " $\mathrm{j}$ ", is known, the estimated coordinates of the target node are calculated according to Eq. 3 and 4 [22].

$$
\begin{aligned}
& \operatorname{maxj}\left(x_{j}-d_{i j}\right) \leq x_{i} \leq \min j\left(x_{j}+d_{i j}\right) \\
& \operatorname{maxj}\left(y_{j}-d_{i j}\right) \leq y_{i} \leq \operatorname{minj}\left(x_{j}+d_{i j}\right)
\end{aligned}
$$

The position detection based on RSSI does not always give reliable results. RSSI values can be affected from a lot of parameters, such as environmental factors, antenna type, the direction of the node, non-homogeneous signal power distribution etc. Since the method is prone to error, the parameters of the environment and nodes should be examined, and necessary calibrations has to be performed carefully 
[15][17][18]. Otherwise, the results obtained could be meaningless. For this, there are several calibration techniques. "Horizontal Calibration" and "Circular Calibration" are some of them. Therefore, obtaining of an empiric equation of RSSIDistance relationship, and the calibration process come into prominence.

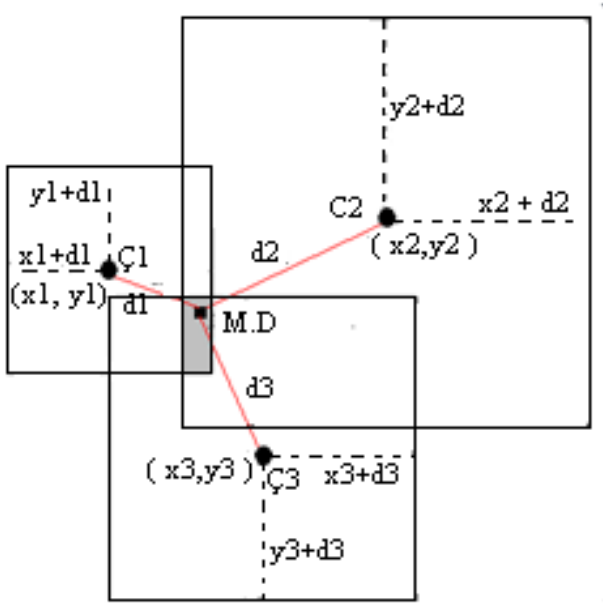

Fig 4: Bounding Box Method

In this study, "Circular Calibration with Offset Value Technique" was used [20]. The technique is an environmentdependent, and it decreases the errors of position detection performed by Bounding Box. In this approach, the results of various experiments achieved by the circular calibration have been improved. The intersection can be increased or decreased by using the offset value. The use of the offset value is completely optional. The equation obtained from "Circular Calibration with Offset Value" is given in Eq. 5. There, "D" represents the distance; "R" denotes RSSI value.

$$
D=e^{-\frac{(R+46.291)}{12.92}}+1.1
$$

\section{SIMULATION OF THE WSN SYSTEM}

TOSSIM was preferred as emulator. As is known, TOSSIM has no a graphic screen. Outputs of a program codes can be follow on a terminal window. So, Tkinter GUI-Toolkit was used for visualizing of the simulation.

The simulation of the system is based on the mathematical model of the indoor place explained above. The coverage area a vehicle was in was calculated by considering the pixel information of it. For the simulation of vehicle guidance, the flow diagram of the Edge-Masking Technique is shown in Fig.5. It was assumed that the vehicles had necessary indicators, such as Up, Down, Ok, 1st. 2nd. 3rd. vertical region etc.

The equation of RSSI-Distance relationship and its graphic are denoted in Fig.6. The equation used in the simulation was obtained from experimental studies [2]. The node codes used in the simulation were written in nesC language. Also these codes were used in programming real nodes. As is known,

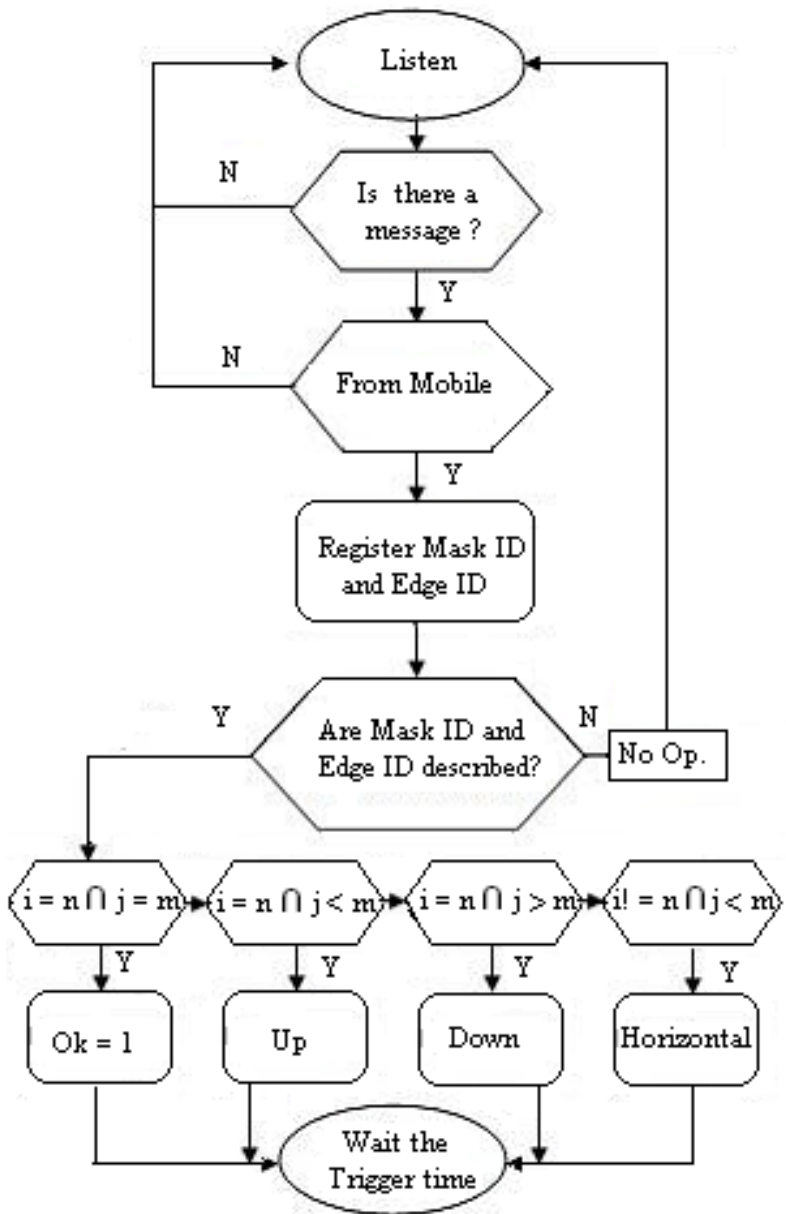

Fig 5: Flow diagram of the guidance control

TOSSIM simulator doesn't have GUI toolkit and it uses a terminal window for outputs. Therefore, Tkinter GUI toolkit was preferred for visualizing of the simulation of the WSN system.

During the simulation, a data-sending to any MON was carried out by "Packet Injection" method of TOSSIM [11]. Thus, an operation of new task-sending from the base station could be simulated interactively. A view of the simulation window is shown in Fig. 7.

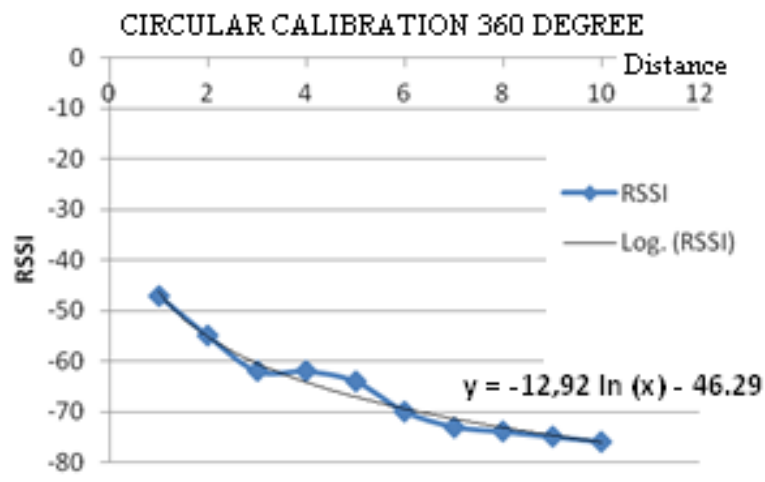

Fig 6: The RSSI-Distance equation and its graphic 


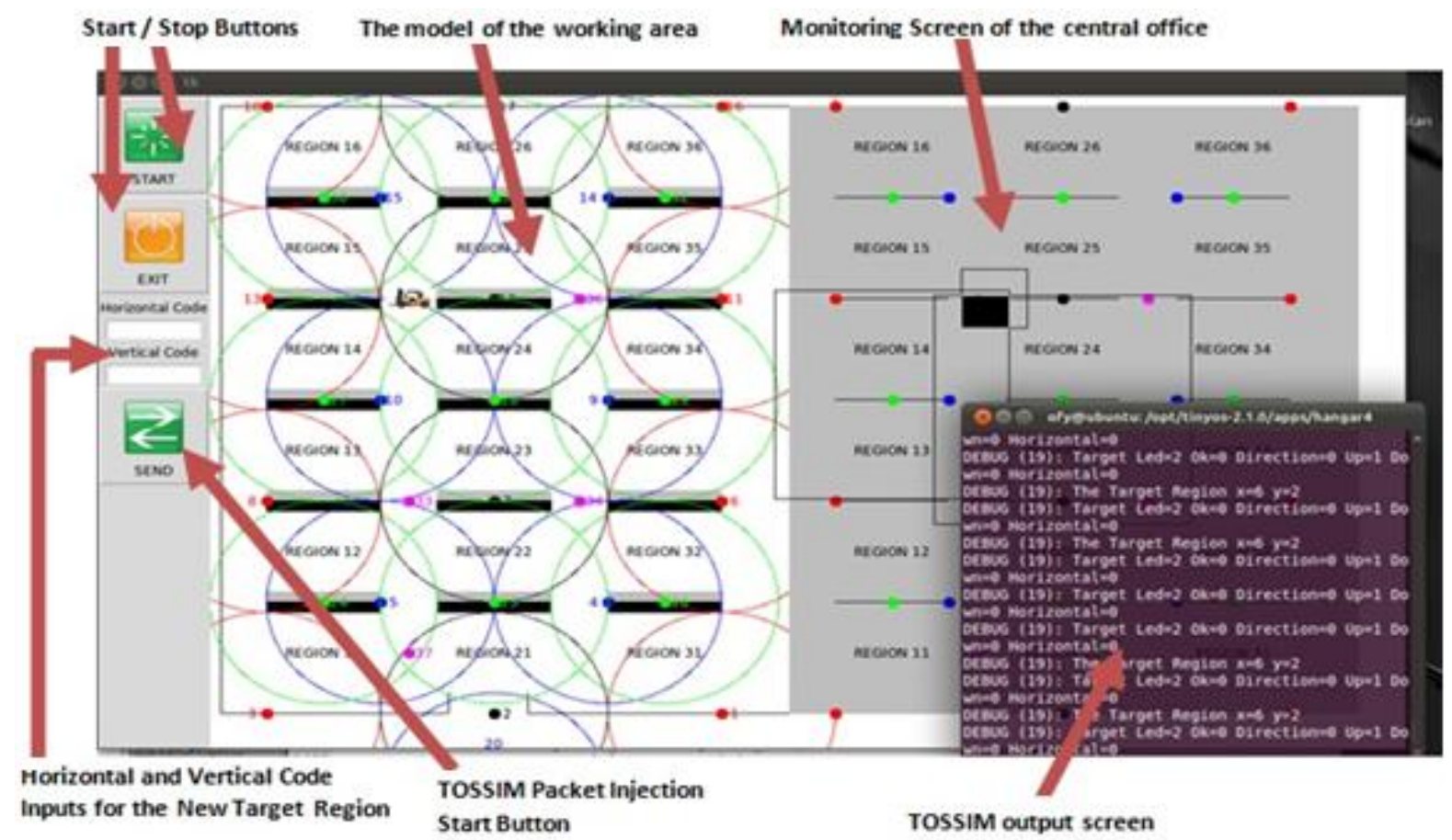

Fig 7: The Simulation Screen

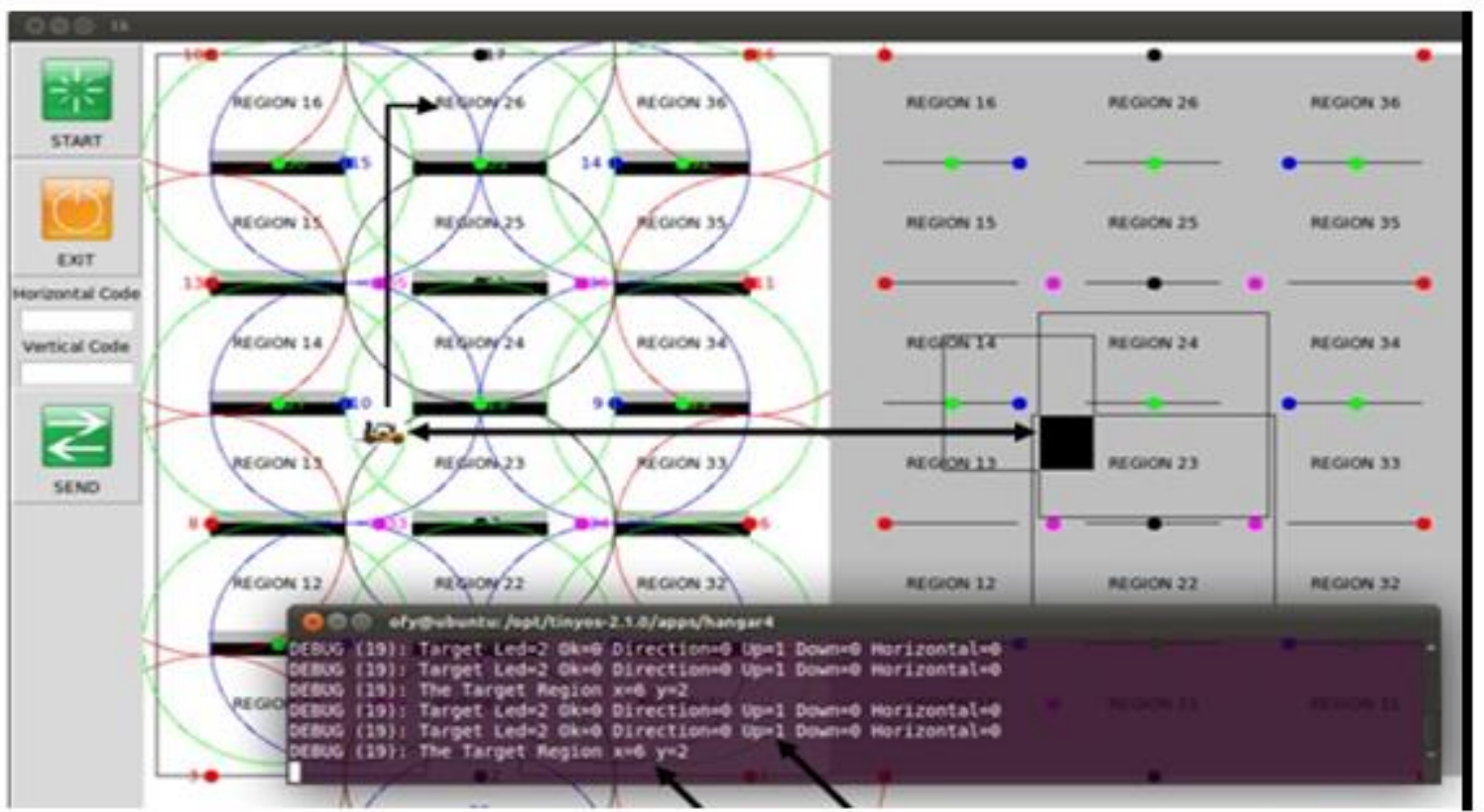

Fig 8: The forklift moving from R21 to $\mathrm{R} 26$

At first, the guidance data of the MON is zero. The MON broadcasts its information obtaining from MNs and ENs by communicating, when its trigger time is expired. The MN will read the message coming from the MON and examine whether the information is described in the look-up table. If described, $(\mathrm{m}, \mathrm{n})$ parameters of the message will be analyzed according to index values. After comparison, guidance information will be loaded into the variables and be broadcasted when its trigger time is expired. The guidance information will be shown via led or screen.
For example, in Fig. 8, a simulation view of the MN receiving the target information "R26" is shown. While the vehicle is moving, the estimated area is demonstrated by a black square in the monitoring window. The nodes the MON will interact are determined according to pixel values. The simulation of the proposed system worked successfully. The next step is a testing process of the system. This implementation was carried out by TelosB nodes using the codes in the simulation. 


\section{IMPLEMENTATION OF THE WSN SYSTEM}

The implementation of the system was carried out according to "Edge Masking Technique" explained in Section 2. Because of the fact that the functions of the nodes are similar, it will be sufficient that the implementation is performed for any one of the regions. The codes used in the simulation were loaded directly into real TelosB TPR 2420 nodes and the implementation was performed successfully.

\subsection{Implementation of the Edge-Masking \\ Technique}

As mentioned before, the MNs were used as decision mechanism. For example, in Fig. 9, a code part of the node " 15 " is shown for region "R25". As indicator, the leds on TelosB were utilized. Therefore, if "horizontal level" is caught, the led of "vertical code" will light. If the MON receives a command "Up", the all leds will turn on and if it receives a command "Down", then the all leds will turn off.

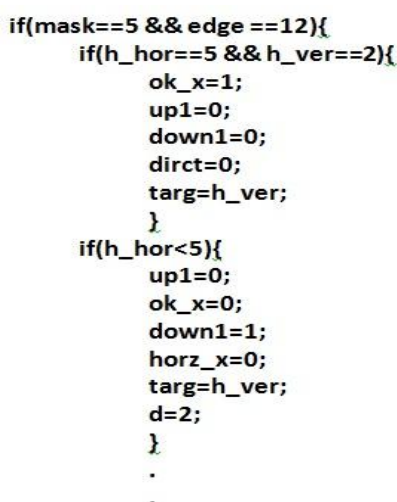

Fig 9: A code part of $M 15$ for $R 25$

A small java application was written in order to send a data packet to the other nodes from Basestation, and it was tried at real time. The interface of the application is shown in Fig.10.

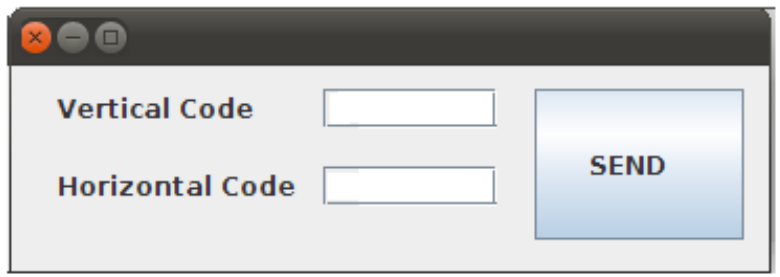

Fig 10: Java program interface to send a new target region

In Figure 11, a state that the MON (the middle node) in "R34" interacts with the MN "9" and the EN " 11 " is shown. In the practical application, the target place (Rij) was changed by data packets sent from Basestation and the status of the leds on the node were watched. For instance, as can be seen in Fig.11.a, if the MON receives a target command for "R24", the led 2 only will light because the horizontal level is caught and it is sufficient to show the vertical code only. In the same way, in Fig.11.b, if a target command "R15" is sent from Basestation, the all leds on the node will light because it is necessary for the MON to move up a top-region. Finally, in Fig.11.c, for target place-command "R11", all leds are turned off because the target place is in two lower-level.

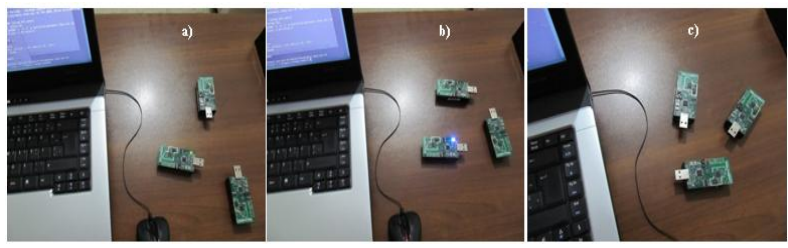

Fig 11: The states of the Leds on TelosB according to the guidance information taken from the mask node a) The horizontal level is ok, b) Move up - All Leds light, c) Move down - All Leds is off

\subsection{Implementation of Position Detection}

The position detection methods explained in Section 2, were applied to a specific closed area, but the implementation could similarly be applied to the entire area. In the experiments, four anchor nodes, a target node and a PC including a basestation node and an application program written in JAVA were used. The application program provided user with both calculation results and visuality. A view of the program interface and the test environment are shown in Fig.12 a,b. The experiments performed earlier show that the circular calibration with offset value technique is more stable than horizontal calibration and circular calibration. According to the all calibration methods, the experiment results for each anchor are displayed in Fig.13 a, b, c, d. As can be seen in the Fig.13, the best results were obtained from the circular calibration with offset value technique [2].

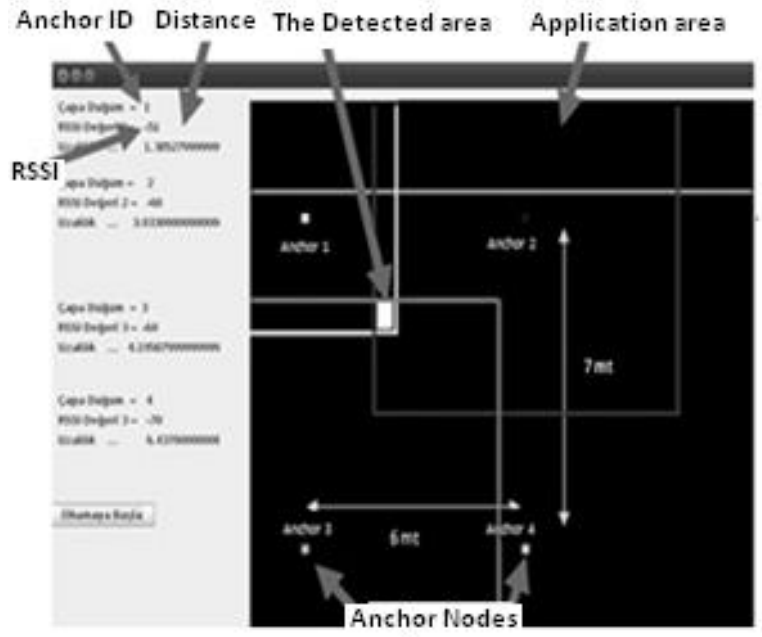

a)

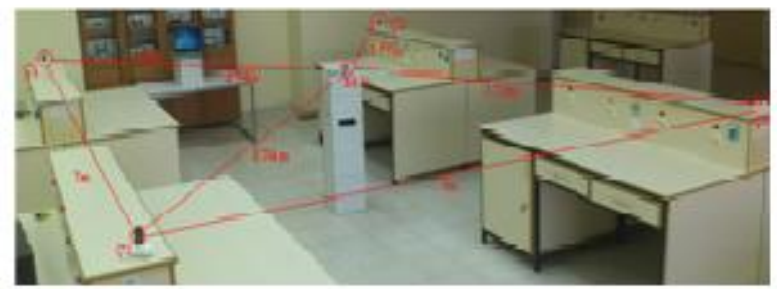

b)

Fig 12: a) A Screenshot of the Java program developed for position detection based on Bounding Box Method b) The test environment 


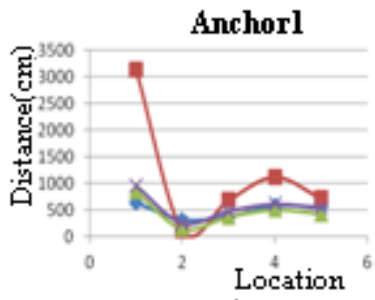

a)

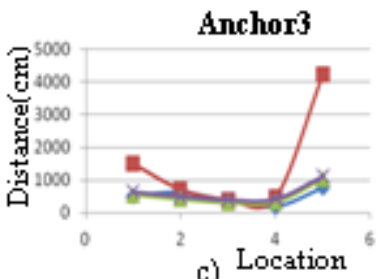

c) Location

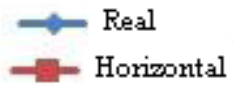

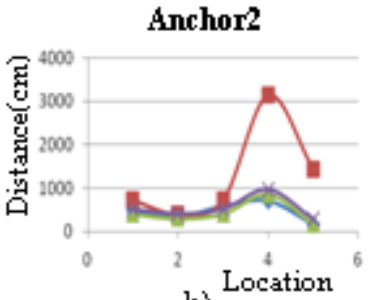

b)

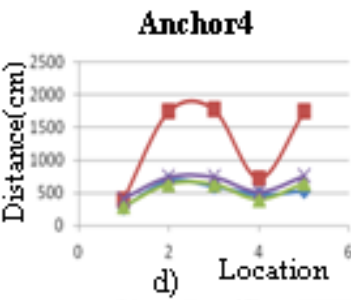

d)
- Circular

$\longrightarrow$ Circular with offset

Fig 13: The approach graphics obtained by different calibration techniques for each anchor nodes

As a real-time screenshot of Bounding Box method using circular calibration is shown in Fig. 14.a, a real-time screenshot of circular calibration with offset value is denoted

in Fig. 14b. As can be seen in the figure, the location of the target node, which could not been detected by circular calibration, could been pinpointed by the circular calibration with offset value.

The example given in Table 4 shows the advantage of the calibration with offset value over circular calibrations. According to Bounding Box equations 3 and 4, for a target node whose coordinate is $(3.52,6.68)$, the estimated coordinate could not be calculated mathematically correctly by circular calibrations. Whereas, it could be calculated consistently by the circular calibration with offset value.

Table 4. A mathematical sample for Bounding Box

\begin{tabular}{|l|c|c|c|c|c|c|}
\hline & Anc & Anc & Anc & Anc & The coord. of the target \\
\cline { 6 - 7 } & $\mathbf{2}$ & $\mathbf{3}$ & $\mathbf{4}$ & $\mathbf{x}$ & $\mathbf{y}$ \\
\hline Real (m) & 3,82 & 2,72 & 6,66 & 6,01 & 3,52 & 6,68 \\
\hline $\begin{array}{l}\text { Circular } \\
\text { Cal.(m) }\end{array}$ & 2,81 & 2,08 & 5,48 & 8,56 & $\begin{array}{l}3,98<\mathrm{x}<2,81 \\
\text { Impossible }\end{array}$ & $5,98<\mathrm{y}<6,68$ \\
\hline $\begin{array}{l}\text { Circular } \\
\text { Cal. With } \\
\text { off. (m) }\end{array}$ & 3,91 & 3,18 & 6,58 & 9,66 & $2,82<\mathrm{x}<3,91$ & $4,82<\mathrm{y}<7,58$ \\
\hline
\end{tabular}

In the other experiments performed, the technique of circular calibration with offset value proved its advantage over circular calibrations.
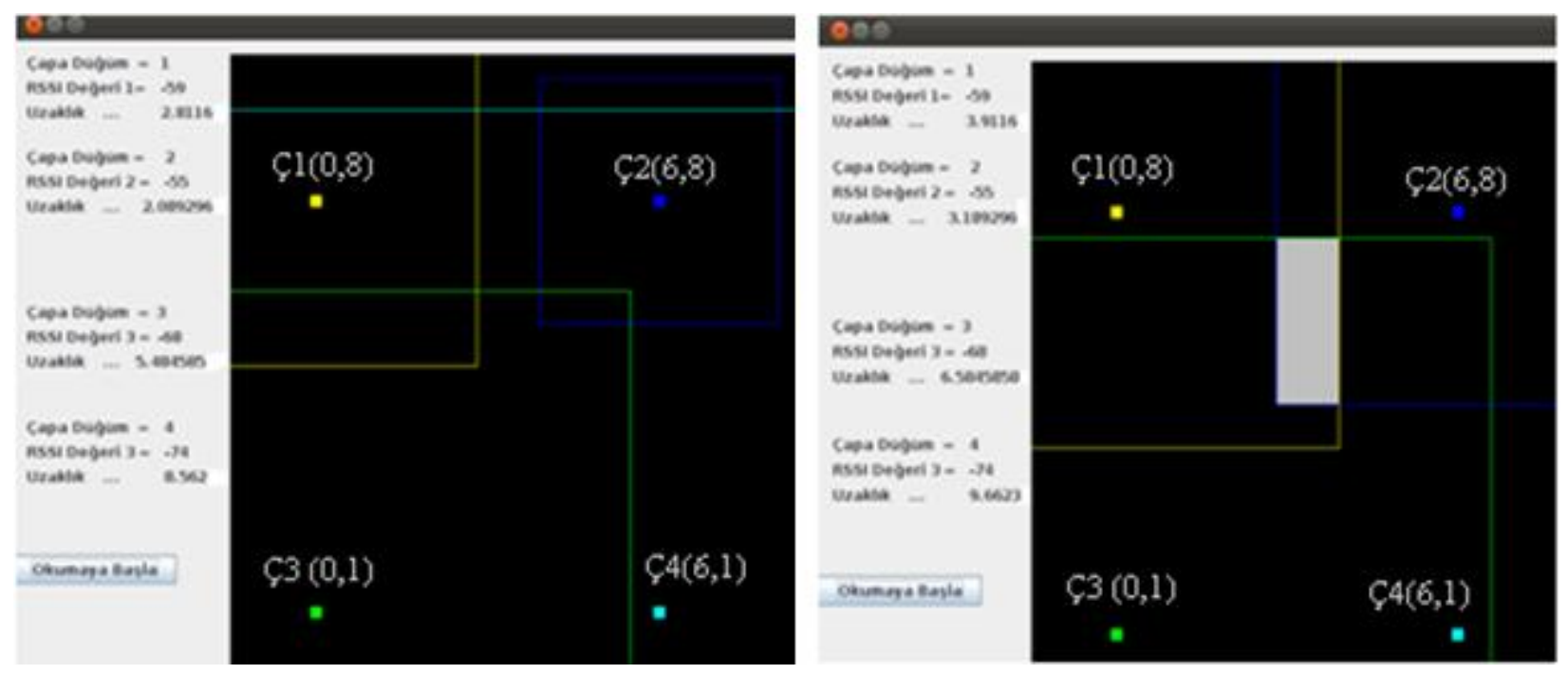

Fig 14: a) Uncertain detection with circular cal.

b) Position detection based on the circular calb. with offset value

\section{CONCLUSION}

In this article, an approach of a low-cost monitoring and guidance system, making use of an existing WSN system deployed for general-purpose in a big closed workplace, was presented. A scenario named "Vehicle Guidance and Tracking in an Indoor Environment" was used in the system. For the system, a technique called "Edge-Masking" was proposed and applied in order to guide vehicles to a target region via the existing WSN. This technique is based on evaluation of a knowledge coming from nodes having different operation modes. This evaluation was carried out by decision-making nodes. Next, the location of a mobile node (MON) was estimated, and then appropriate guidance commands were sent to the vehicles. Bounding Box Method was preferred as position detection method. For the purpose of achieving an equation of RSSI-Distance relationship, experimental studies were carried out by TelosB nodes in an indoor place. The calibration of nodes was made by an improved technique, "Circular Calibration with Offset Value". With the technique, errors in Bounding Box method decreased. The simulation of the proposed system was carried out by TOSSIM. The results of both the implementation and the simulation demonstrated the success of the proposed system. 


\section{ACKNOWLEDGMENTS}

This Study Was Supported By The F.U. Project, FubapMf.11.42.

\section{REFERENCES}

[1] Akyildiz, I., Su, W., Sankarasubramaniam, W., Çayirci, E., 2002, Wireless Sensor Networks: A Survey, Elsevier Computer Networks, March, volume 38, Issue 4, pp. 393-422,

[2] Yildirim, G. ,2012, Position Detection in Wireless Sensor Networks. Master Thesis, Firat University, Elazig, Turkey

[3] Verbaendert, N., Henderickx, D., Doggen, J., 2008, Emote: A Wireless Sensor Network Monitoring Tool With Node Localization Using RSSI, Papers of e-Lab, Master Theses, The University Collage of Antwerp, Belgium

[4] Santos, F. 2008, Localization in Wireless Sensor Networks, ACM Journal Name, Vol. V, No. N, November, pp. 1-19.

[5] Potdar, V., Sharif, A., Chang, E., 2009, Wireless Sensor Networks: A Survey, Advanced Information Networking and Applications Workshops, WAINA '09. 21

[6] Willig, A. 2006. Wireless Sensor Networks: Concept, Challenges and Approaches. In e \& I Elektrotechnik und Informationstechnik. Vol. 123. Springer Wien, 224\{231.

[7] Kim, S. J., Seo, J. H., Krishna, J., Kim, S.J.,2008, Wireless Sensor Network based Asset Tracking Service, PICMET 2008 Proceedings, pp: 2643,2647- July, Cape Town, South Africa,

[8] Kamath, S,. Meisner, E., Isler, V., 2007, Triangulation Based Multi Target Tracking with Mobile Sensor Networks, 2007 IEEE International Conference on Robotics and Automation, pp.3283-3287, Roma, Italy, April

[9] S. Mahlknecht, S. Madani, 2007, On Architecture of Low Power Wireless Sensor Networks for Container Tracking and Monitoring Applications", Proceedings of IEEE INDIN07, pp:-353-358,

[10] G.-y. Jin, X.-yi Lu, and M.-Soon Park, 2006, Dynamic Custering for Object Tracking in Wireless Sensor
Networks in Ubiquitous Computing Systems, pp.200209.,

[11] www.tinyos.net, 1.2. 2012

[12] BI Yan-Zhong, SUN Li-Min, ZHU Hong-Song, YAN Ting-Xin, LUO Zheng-Jun A, 2006, Parking Management System Based on Wireless Sensor Network, ACTA AUTOMATICA SINICA, November, Vol. 32, No.6

[13] Idris M. Y. I., Tamil E. M., N., Noor M. and K. Fong W., 2009, Parking Guidance System Utilizing Wireless Sensor Network and Ultrasonic Sensor, Information Technology Journal, ISSN 1812-5638.

[14] Anna Ha'c, Wireless Sensor Network Designs, 2003 , University of Hawaii at Manoa, Honolulu, Publisher: Jhon Wiley \& Sons, USA

[15] Baunach M., M"uhlberger C., 2009, Analysis of Radio Signal Parameters for Calibrating RSSI Localization Systems, Technischer Bericht Nr. 455

[16] Yoon L.S., Ghosh, A., Intelligent Parking Lot Application Using Wireless Sensor Networks, Proceedings of IEEE conference, 978-1-4244-2249-4/08

[17] Fang, Z., 2010, RSSI Variability Characterization and Calibration Method in Wireless Sensor Network, Information and Automation (ICIA), IEEE International Conference, pp. 1532-1537,

[18] Aguilar, L., Licea, G., García. M, J. A.,2011, An Experimental Wireless Sensor Network Applied in Engineering Courses", Comp. Appl. Eng. Educ., 19: Page : 777-786.

[19] Ayna, M. A., Alkan F. 2010, Telsiz Duyarga Ağlarında Alınan İşaret Gücü Göstergesi (RSSI) İle Konum Belirleme Ve Haritalama ,http://www.emo.org.tr/ekler/ 203890ee8233a1a_ek.pdf, 20 Mart 2012

[20] Yildirim,G., Tatar,Y., 2012, Enhancement Of The Empiric RSSI - Distance Relationship By Experimental Datum In Wireless Sensor Networks, IADIS Int. Conf Applied Computing, pp:380-384,Madrid,Spain,

[21] http://www.memsic.org, TelosB Datasheet, MEMSIC

[22] Whitehouse C.D., 2002, The Design of Calamari: an Adhoc Localization System for Sensor Networks, Research Project, University of California at Berkeley 